\title{
Investigation and Modelling of the Progression of Zinc Leaching from Large Sphalerite Ore Particles
}

\author{
${ }^{*}$ Yousef Ghorbani ${ }^{1,2}$, Jochen Petersen ${ }^{1,2}$, Megan Becker ${ }^{1}$, Aubrey N. Mainza ${ }^{1}$ and Jean-Paul Franzidis ${ }^{1}$ \\ ${ }^{1}$ Minerals to Metals Signature Theme, Department of Chemical Engineering, University of Cape Town, Private \\ Bag X6, Rondebosch 7701, South Africa. \\ (*Corresponding author: yousef.ghorbani@uct.ac.za) \\ ${ }^{2}$ Centre for Bioprocess Engineering Research (CeBER) Department of Chemical Engineering, University of Cape \\ Town.
}

\begin{abstract}
X-ray Computed Tomography (CT) was used to follow the progression of $\mathrm{Zn}$ leaching in a number of individual sphalerite ore particles, which were subjected to a long-term simulated heap bioleaching environment. The ore was prepared by two different modes of comminution - HPGR at 90 bar and cone crusher - and individual particles were selected from three different size fractions. Investigation of the reacted fraction of $\mathrm{Zn}$ vs distance from the centre of each particle indicated that leaching from large particles leads to near complete conversion near the surface, but only partial conversion in the zones that are closer to the centre of particles. The cores of the cone-crushed particles show hardly any conversion at all, especially in the larger particle sizes.

Mathematical analysis shows that leaching from the large particle does not follow the shrinking core model. It is shown that the progression can rather be described by a combined reaction-diffusion process progressing through the network of cracks and pores closer to the particle surface. Extent and depth of this network are a function of particle size and comminution method. A simplified rate model is proposed that describes the extent of leaching as a function of time in terms of a set of parameters that can all be related to just particle size and crushing mode.
\end{abstract}

Key word: large particles, mineral conversion, shrinking core model, heap leaching. 


\section{Introduction}

Most heap bioleaching models, regardless of whether they deal with leaching at the particle scale (Braun et al., 1974; Bartlett, 1992; Davis and Ritchie, 1987; Roman, 1974; Shafer et al., 1979) or focus on bulk scale phenomena, such as liquid flow, gas flow, and temperature distribution (Dixon, 2000; Dixon and Petersen, 2003; Leahy et al., 2007; Moreno et al., 1999; Ogbonna et al., 2006; Petersen and Dixon, 2002; Pantelis et al., 2002; Sidborn et al., 2003), account for the effect of particle topology by using simplified models such as the shrinking core concept applied to an average particle size or a generic size distribution.

Implicit in these simplifications is an assumption regarding the relative significance of particle scale phenomena and which kinetics (diffusion or reaction) are limiting at the particle scale. Yet it must be understood, that a conventional shrinking core approach would work only for ore particles that are homogeneously porous and have mineral grains well distributed throughout (Liddell, 2005; Velardo et al., 2002; Vegliò et al., 2001). The shrinking core model has been shown to be a poor predictor of leach kinetics based on the crushed ore size distribution (Miller, 2003). However, the model itself is a very useful tool to analyse commercial heap data. Data is obtained from batch leaching, which is then fitted according to the equations describing the topological model, and then the set of parameters that gives the best fit is chosen as best representative of the system. There are no reliable criteria to predict a priori the rate-controlling regime in which a given system will operate. Clearly, obtaining these criteria will require a greater understanding of the controlling processes on a microscopic level (Liddell, 2005; Velardo et al., 2002).

A part of the difficulty in understanding leaching reactions lies in their heterogeneous nature and the variability from one ore or concentrate to another. However, another aspect relates to the fact that the conventional shrinking-core equations have been narrowly focused on the lixiviant and the particles being leached (Liddell, 2005). Models usually assume spherical geometry of the particles, although ore or concentrate particles are never spherically symmetric. Deviation from the spherical shape leads to an increase in the surface-to-volume ratio (Rossi, 1990). Furthermore, particle scale effects in heap bioleaching are influenced by other factors, including the ore porosity, grain exposure, grain and particle size and shape distribution, the ore mineralogy, surface properties, pore size distribution and microbial interactions (Ghorbani et al., 2011a).

The overall rate at which a mineral is dissolved from an ore particle is quite often the manifestation of a complex network of individual phenomena, each proceeding at its own intrinsic rate. For finely ground particles such as those encountered in tank leaching processes, leaching rates are typically 
controlled by the intrinsic kinetics of mineral breakdown. In heap or dump leaching, on the other hand, mineral grains are usually embedded within larger ore particles or solution-filled agglomerates, and thus accessible only by diffusion through a network of pores (Bouffard and Dixon, 2001).

Compared with the efforts usually taken to determine and interpret the effects of temperature, reagent concentration and other solution variables, very little has been done to measure or detect the effect of the variables relating to the actual ore particles in most systems. Historically this has been constrained by technical limitations to study mineralogy and the inability to look 'inside' particles while they were leaching. Although such limits in the techniques for measurement of these parameters remain, an increasingly wider range of microscopy and tomography techniques is available to provide 'visual' evidence for suspected and unsuspected phenomena. In this paper one of these techniques, $\mathrm{X}$-ray Computed Tomography (X-ray-CT), is used to explore the progression of leaching inside particles.

In a previous paper (Ghorbani et al., 2012a) we have described the raw results of a leaching study in which particles of a sphalerite ore at different particle sizes and prepared by different comminution methods were exposed to a bioleaching environment over an 11-month period. In the present study, we apply X-ray CT as an advanced diagnostic and non-destructive technique for measuring the presence and disappearance of mineral grains within individual ore particles in the course of the long-term leach experiment.

\section{Materials and Methods}

A bulk sphalerite ore (see Table 1 for main sulphide minerals) was obtained from the Gamsberg Zinc mine in the Northern Cape Province, South Africa. The bulk sample was initially crushed by a jaw crusher from $300 \mathrm{~mm}$ top size down to $-40 \mathrm{~mm}$. The jaw crusher product was representatively split into $250 \mathrm{~kg}$ bags for further crushing down to $-25 \mathrm{~mm}$ by either a Köppern HPGR unit at 90 bar pressure or cone crusher at Mintek in Randburg, South Africa. Products from the cone crusher and HPGR were then screened into five size fractions $(+23 /-25,+16 /-23,+14 /-16,+6.75 /-14,+5.25 /-6.75$ $\mathrm{mm})$. In this study, subsamples from the large size fraction $(+23 /-25 \mathrm{~mm})$, medium size fraction $(+14 /-16 \mathrm{~mm})$ and small size fraction $(+5.25 /-6.75 \mathrm{~mm})$ were subjected to a long-term bioleach study in a specially designed circulating fluid fixed-bed reactor. Results of this study are described in detail in a previous paper (Ghorbani et al., 2012a). 
Table 1: Mineralogical composition of the ore

\begin{tabular}{|l|c|}
\hline Mineral & Amount (Wt \%) \\
\hline Pyrrhotite & 1.2 \\
\hline Pyrite & 33.8 \\
\hline Sphalerite & 16.0 \\
\hline Galena & 0.2 \\
\hline
\end{tabular}

In the present study, we describe an analysis of the progress of leaching in selected individual particles from different size fractions in the course of the previous bioleaching study. For this, the reactors were stopped from time to time to investigate the progress of leaching by taking and then analysing X-ray CT images of the same individual particles. The use of this technique to identify both cracks and sphalerite grains within a matrix of gangue and other sulphide minerals especially pyrite, has been discussed in a previous paper (Ghorbani et al., 2011b). This approach was used here to collect images of particles before and at different stages of the leach experiment.

An HMXST CT scanner (X-Sight X-ray Services in Stellenbosch, South Africa), with 225 kV X-ray source, $3 \mu \mathrm{m}$ resolution reflection target, and interchangeable Nano-tech $1 \mu \mathrm{m}$ transmission target was employed. Further operating details of the X-ray CT conditions are given in Table 2.

Some calibration was needed in order to be able to identify the optimal measurement settings to differentiate between different phases (cracks and the various minerals present in the ore particles), given that there is some overlap in the grey level brightness. In practice, density measurement from X-ray tomography data can be obtained either by calibrating the CT machine with objects of known density and obtaining a correlation that relates density with the attenuation coefficient, or by using a dual energy scanning scheme to determine directly the density of the material (Ghorbani et al., 2011b). In this study, the dual energy scanning method was used for calibration, as it allows one to determine both the density and the effective atomic number of the object of interest separately. Thus, the sensitivity of the analysis is significantly increased. Following the reconstruction of the acquired X-ray CT data, the ore particles were analysed using the VGStudio Max 2.1 image analysis software (Volume Graphics GmbH, Heidelberg, Germany). The crack depth was calculated from analysing the initial X-ray CT images of the particles at the scale of image resolution (up to $15 \mu \mathrm{m}$ ). 
Table 2: Experimental conditions used for X-ray CT measurements.

\begin{tabular}{|l|l|}
\hline X-ray energy & $130 \mathrm{kV}$ and $200 \mathrm{kV}$ micro-focus source with $3 \mu \mathrm{m}$ focal spot size \\
\hline X-ray intensity & $120 \mu \mathrm{A}$ beam current \\
\hline Integration frames & Each projection image was exposed for 1 second per frame - no integration of frames \\
\hline Random movements & No random movements and continuous rotation of sample \\
\hline Beam hardening filters & $\begin{array}{l}\text { A software correction was used which modifies the Beer-Lambert Law (attenuation } \\
\text { curve) to match the sample. A pre-set correction was chosen including both linear } \\
\text { and quadratic curve fitting }\end{array}$ \\
\hline Second radiation filters & $1 \mathrm{~mm} \mathrm{Cu}$ \\
\hline
\end{tabular}

The results were further validated with those obtained using traditional (destructive) techniques such as SEM/EDS and QEMSCAN on other particles taken from the same reactor at the various intervals.

Only few selected particles from each size fraction (eight particles for the small, four for medium and two for large size fraction) were analysed using X-ray CT, and are hence not necessarily representative of the overall leach trends. To ensure that results from the analysis of the individual particles compare to those of the overall leach experiments, total $\mathrm{Zn}$ conversions have been compiled from a succession of X-ray CT analyses. These data points are included in Figures 1 and 2 and can be seen to follow the overall solution leach curve in trend, but not necessarily in extent.

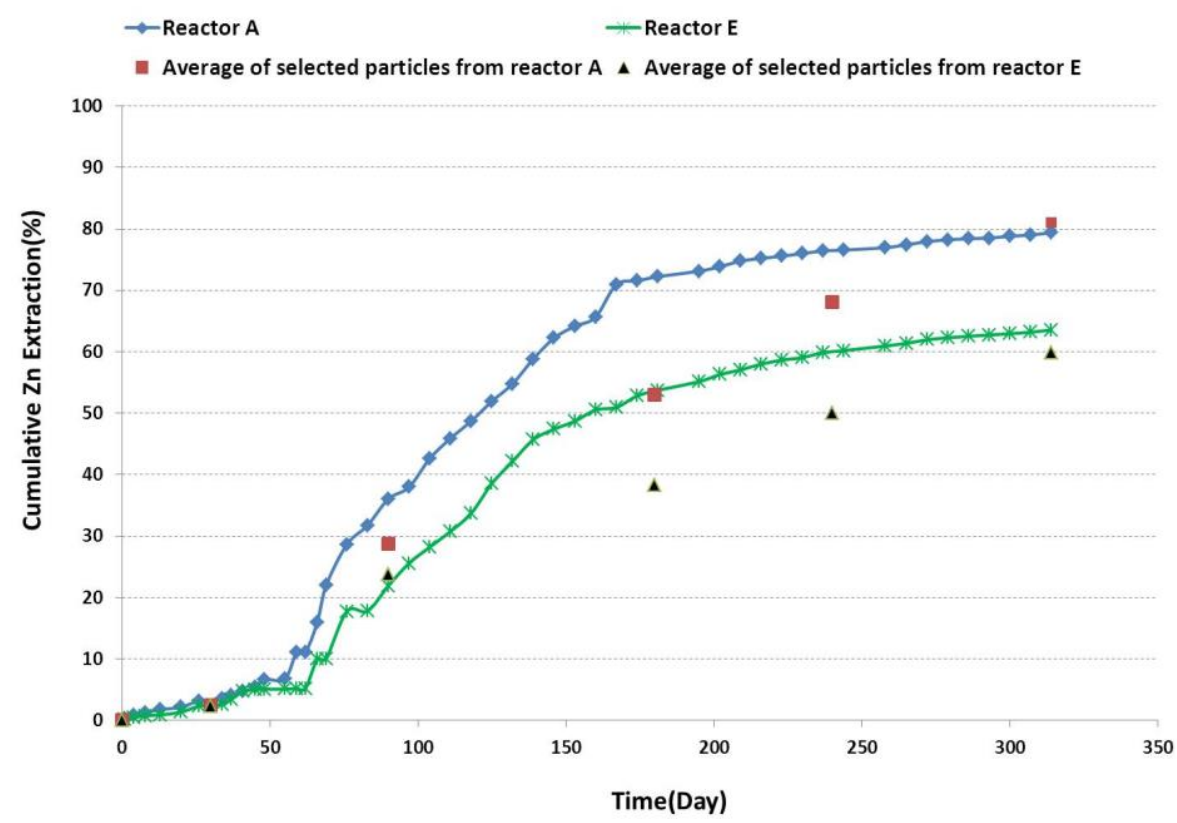

Figure 1: Cumulative amount of zinc in the leach liquors from the reactors A (HPGR-95bar-small size fraction) and $\mathrm{E}$ (cone crusher-small size fraction) (Ghorbani et al., 2012a). Note that the marker points are average $\mathrm{Zn}$ extractions based on the X-ray CT analysis of a small number of selected particles from each leach reactor. 

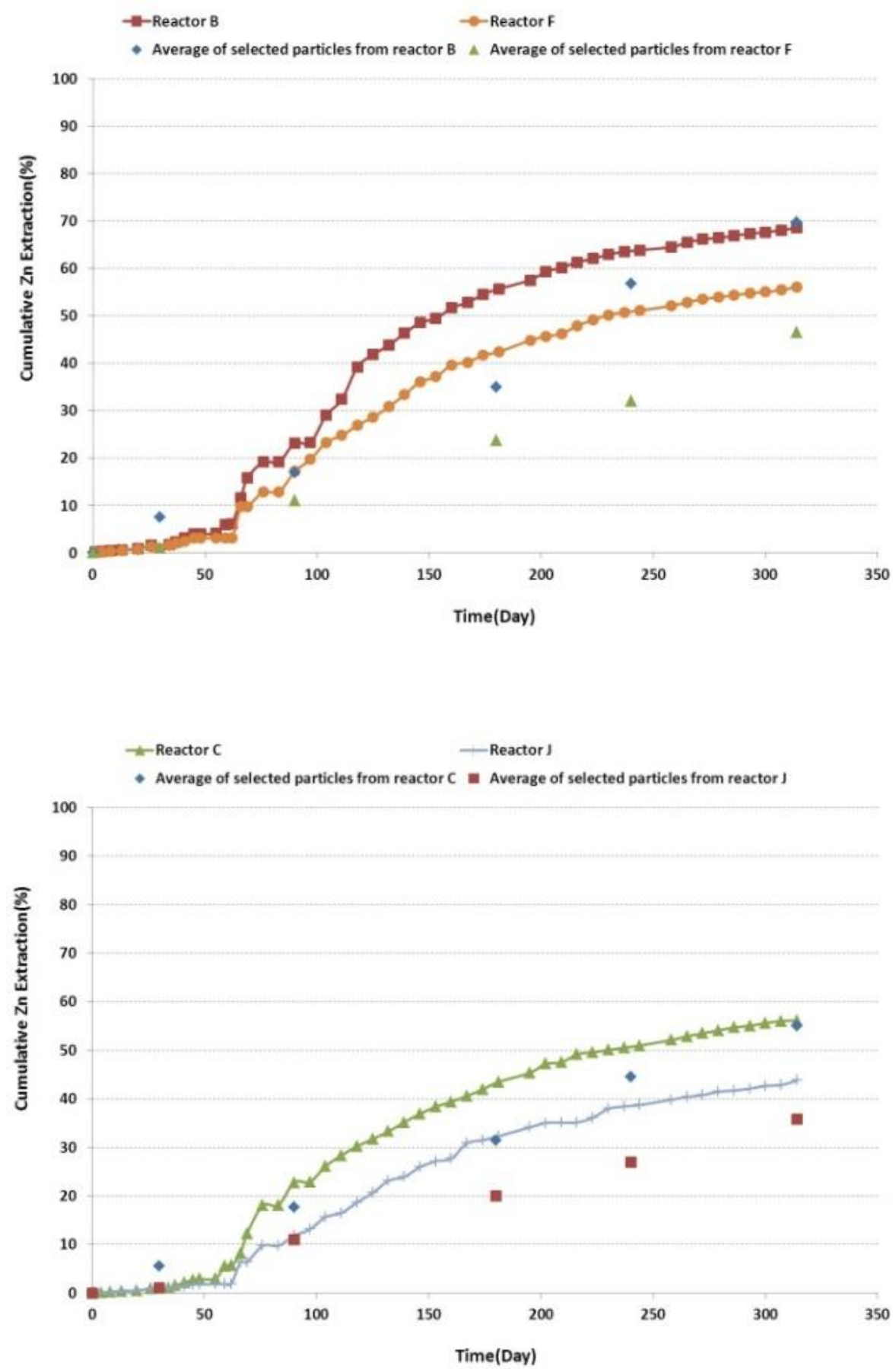

Figure 2: Comparison of the cumulative amount of zinc in the leach liquors; a. reactor B (HPGR-95bar-Medium size fraction) and $F$ (cone crusher-Medium size fraction), b. reactor C (HPGR-95bar-large size fraction) and J (cone crusher-large size fraction). Note that the markers point are average of $\mathrm{Zn}$ extraction related to the selected particles from each leach reactors calculated using X-ray CT.

The graph shows that Zn conversion data obtained from X-ray CT on the solid particles is lower. The fact is that the calculation method when considering the individual particles is not explicit. It is by averaging the zinc grade diminution rate in the selected particles. 


\subsection{Mineral conversion from single ore particles}

To investigate the progression of leaching on the inside of the individual particles, each single selected particle was divided into a succession of shells relating to the distance from the centre of particle, as illustrated in Figure 3. The concentration of $\mathrm{Zn}$ grains were determined in each shell and each time the particle was analysed, thus allowing determination of the progression of $\mathrm{Zn}$ leaching with both time and distance from the surface.

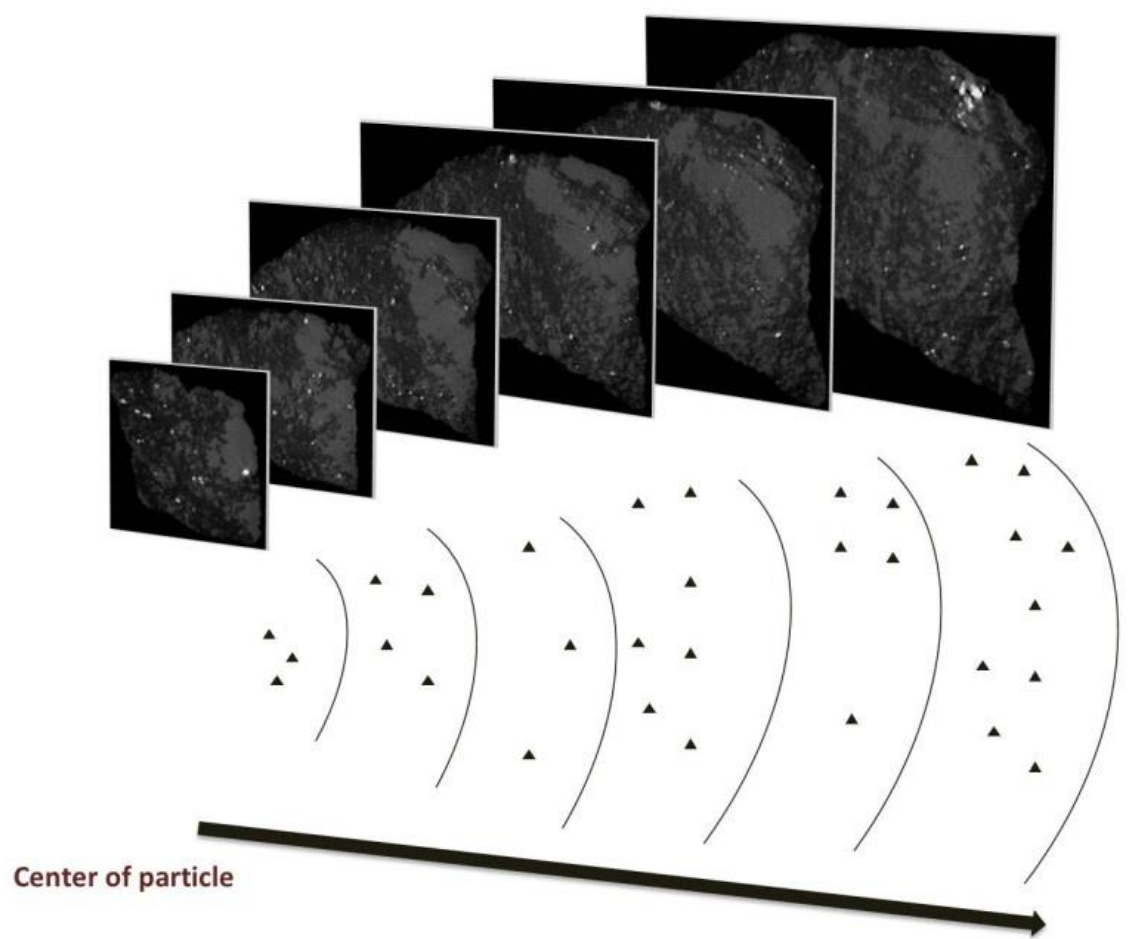

Surface of particle

Figure 3: An illustration of the division of each single particle into different shells bases on the distance from centre of the particle.

Using VGStudio MAX, each shell is defined as a Region of Interest (ROI), and due to the irregular shape of particles, the polygonal surface model tool was used to draw the boundary of each shell following the shape of each particle. Defining thus the ROI in each, $x, y$ and $z$, direction of the image and extracting it with the polygonal surface extraction tool provided by the software, analysis of each sub-zone or shell as a separate 3-D object became possible. Figure 4 shows the average $\mathrm{Zn}$ grain distribution and $\mathrm{Zn}$ grade within selected particles from each size fraction crushed by HPGR at 95bar. Zn percentage in Figure 4 refers to mass percentage of $\mathrm{Zn}$ grains located in a given shell. 
Given that the volumes of the shells are not the same, but become smaller in the centre of the particles, similar percentages of $\mathrm{Zn}$ in each shell translate into different zinc grades (or concentrations). As can be seen, counter to a key assumption for using the shrinking core approach, the sphalerite mineral grains are not homogeneously distributed, but in the present case tend to be concentrated in the particle centre. Figure 5 shows the average $\mathrm{Zn}$ conversion as a function of distance from the centre of the particle in the selected ore particles, produced by the two different comminution devices, and in the different size fractions. This analysis clearly shows that leaching from large particles approaches complete conversion near the surface, but only partial conversion in the zones that are closer to the centre of particles. The cores of the cone-crushed particles show hardly any conversion at all, especially in the larger particles. 

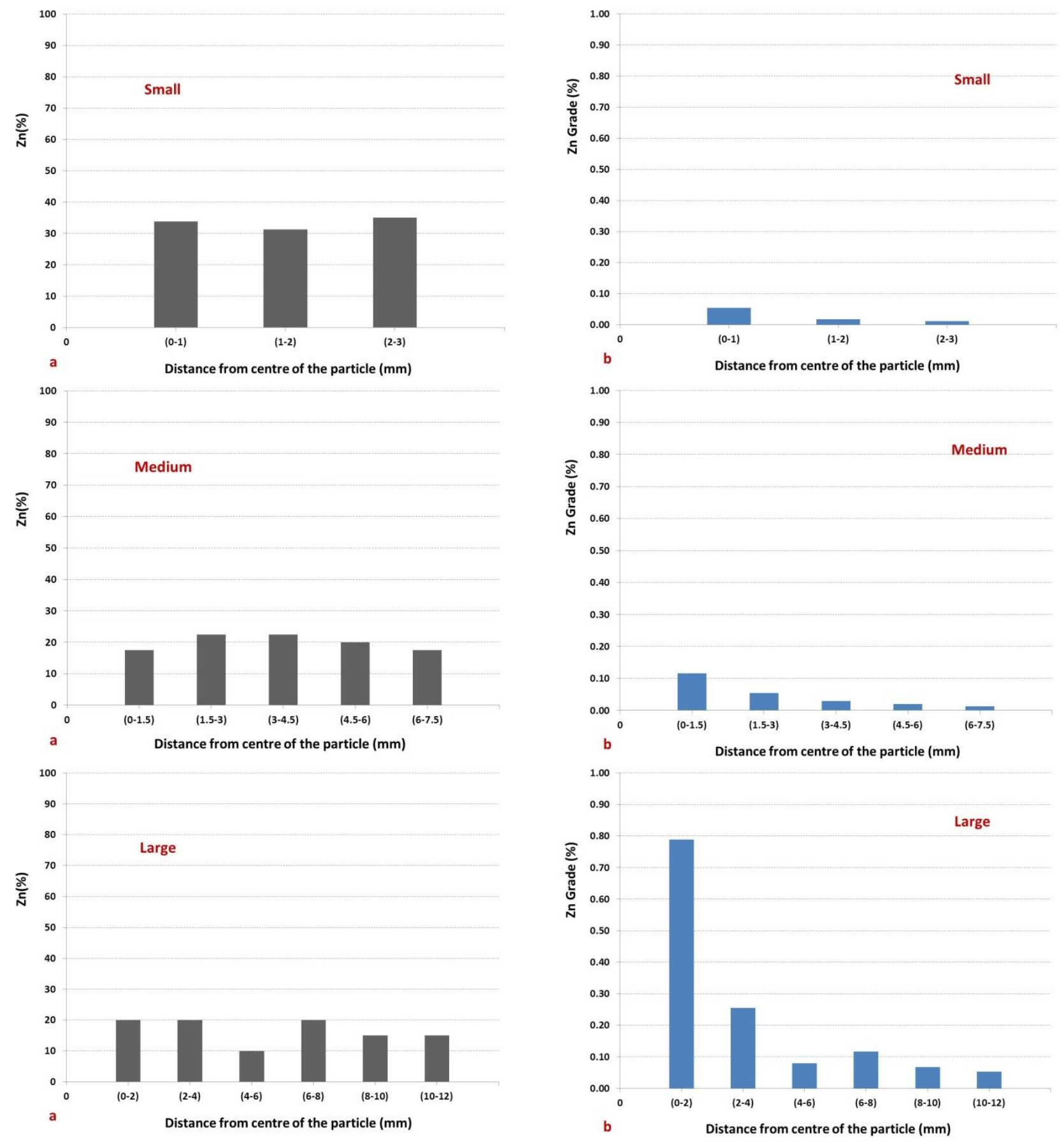

Figure 4: Average $\mathrm{Zn}$ grain distribution (a), and average of $\mathrm{Zn}$ grade (b) within selected particles from each size fraction crushed by HPGR-95bars. 
HPGR
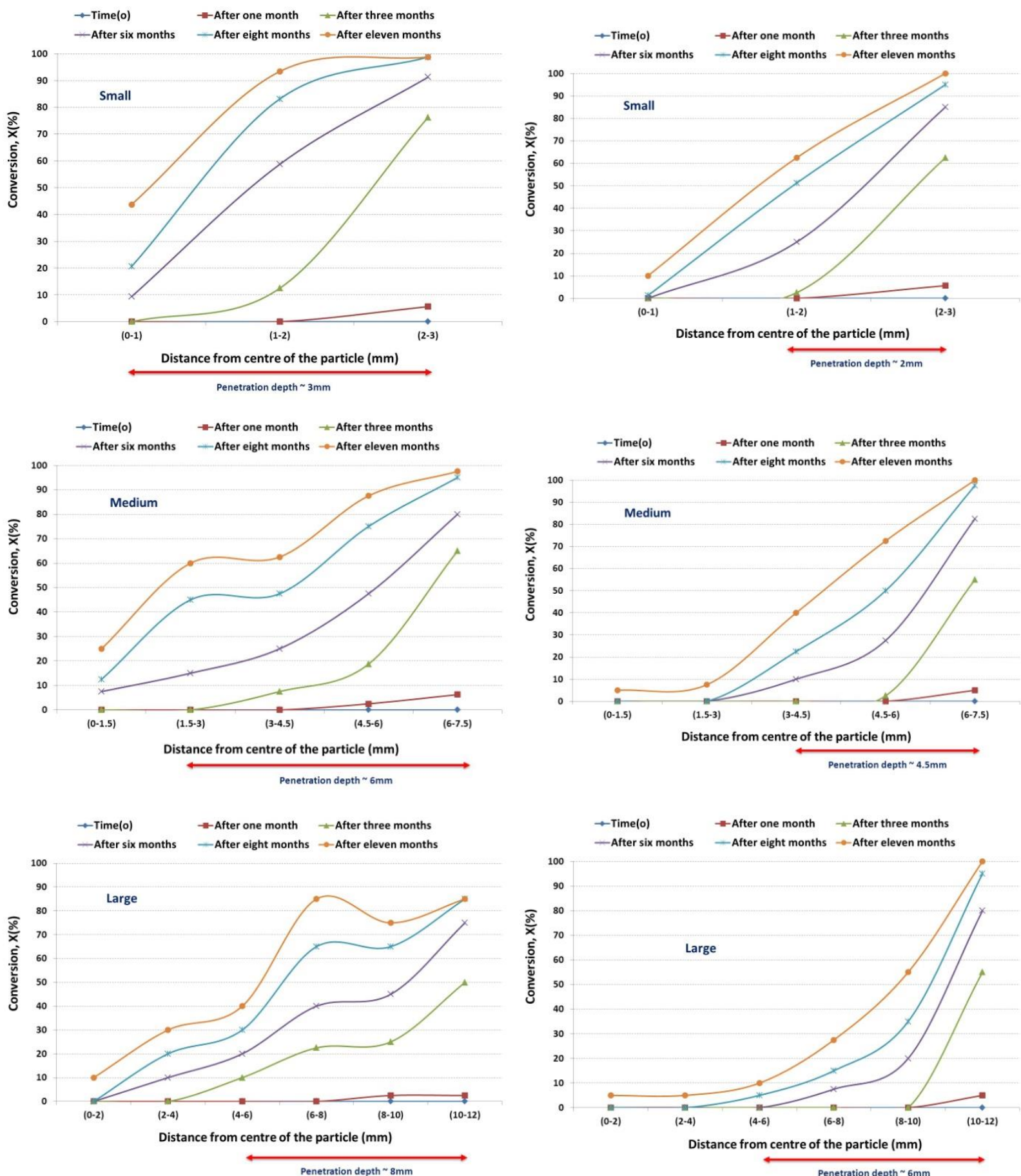

Figure 5: Comparison of the average conversion $X(\mathrm{Zn})$ over time (from start of the experiment) at different positions within an ore particle crushed by HPGR-95bar and cone crusher for different size fractions. Note the deeper penetration in the particle prepared by HPGR.
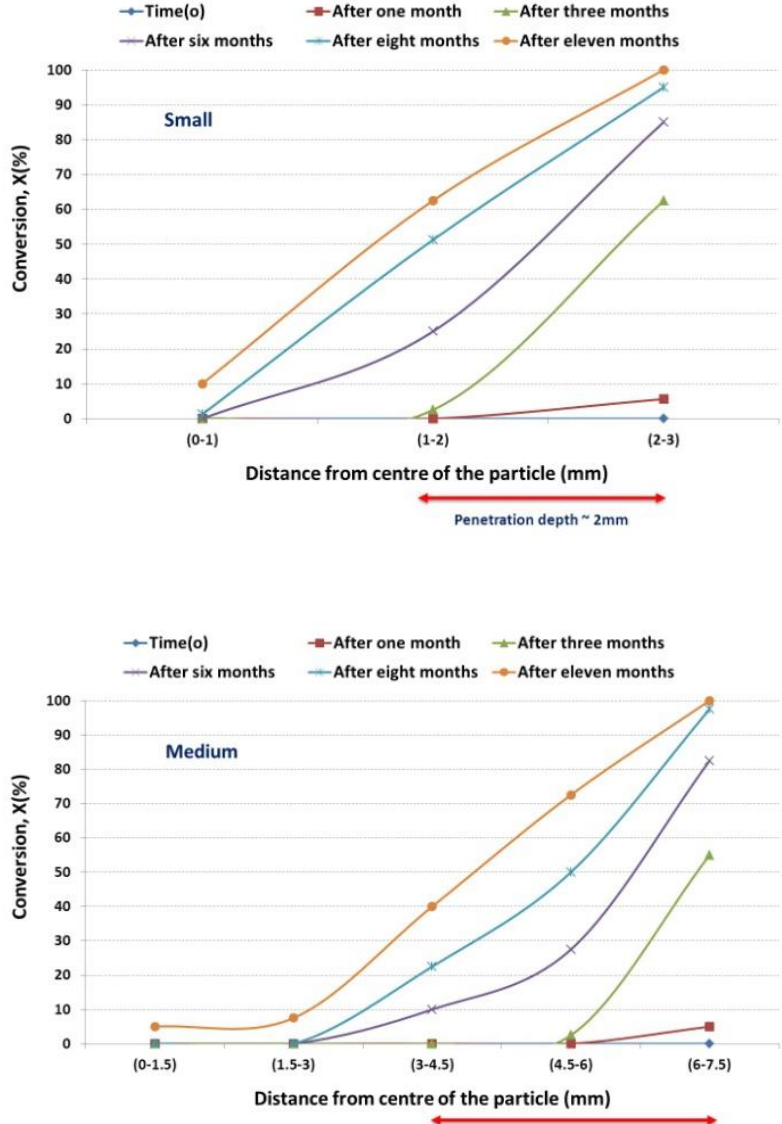

Penetration depth $\sim 4.5 \mathrm{~mm}$ 


\subsection{Crack depth and surface zone}

The higher crack density in the particles crushed by HPGR compared to those produced by the cone crusher (Ghorbani et al., 2012b) provides a larger initial surface zone for reaction. In the HPGR product, the reaction zone covers both the particle surface and subsurface zone, which has access to the surface through cracks. In some particles, even grains close to the centre of the particles dissolved early if they were connected to particularly deep cracks. In the case of the cone crusher product, the outer surface of the particle is the main reaction surface and reaction occurs in the subsurface zones to not nearly the same depth as the particles crushed by HPGR. This implies the existence of micro-cracks in these particles, which were not detectable by the visual techniques used in the present study. Reaction near the surface of particles leads to partial overall conversion in the coarse particles. After this initially rapid phase, leaching slows down dramatically. This is explained by the fact that after depletion of the easy-to-leach minerals near the surface, reagents have to migrate deeper into the particles via the pore network. This will result in a rim-leaching effect in larger particles. All minerals near the surface are depleted first before the leach front can migrate further into the particle. On the inside of the particle, the rate is then controlled by the rate at which reagent (ferric iron in the present case) diffuses deeper into the particle.

Figure 5 clearly shows that HPGR-crushed ore leached more rapidly than the cone-crushed material in all particle size classes, and to slightly greater depth. Table 3 shows the penetration depth $\left(P_{d}\right)$ estimated from Figure 5, as well as average crack depth from the surface of the selected particles of different size fraction as a function of the crushing device.

Table 3: Estimated penetration depth $\left(P_{d}\right)$ and average crack depth of the selected particles after crushing in different size fraction (ND denotes not detected).

\begin{tabular}{l|c|c|c|c|c}
\hline \multirow{2}{*}{ Size fraction $(\mathbf{m m})$} & \multirow{2}{*}{ Mean $\mathbf{R}$} & \multicolumn{2}{|c|}{ Average crack depth $(\mathbf{m m})$} & \multicolumn{2}{c}{ Penetration depth $\left(\mathbf{P d}_{\mathbf{d}}\right)(\mathrm{mm})$} \\
\cline { 3 - 6 } & & cone crusher & HPGR & cone crusher & HPGR \\
\hline Small (-6.75+5.25) & 3 & ND & 1.08 & 2 & 3 \\
Medium (-16+14) & 7.5 & ND & 3.43 & 4.5 & 6 \\
Large (-25+23) & 12 & ND & 6.35 & 6 & 8 \\
\hline
\end{tabular}

It is interesting to note that measured crack and inferred penetration depths for HPGR consistently differ to the order of around $2 \mathrm{~mm}$. This is an indication that cracks detectable by X-ray CT are generally larger than those needed for reagent penetration during leaching. It is therefore safe to 
say that also cone-crushed particles exhibit a network of micro-pores near the surface, even though they are not detectable by X-ray CT. By extension, it is likely that also HPGR crushed particles have a network of such micro-pores deep inside the particles extending beyond the rim of larger pores detectable by X-ray CT.

The results presented in Figure 6 indicate furthermore that the penetration depth $\left(P_{d}\right)$ is as function of both the comminution mode and the particle size in terms of radius $(R)$. It is clear that prevalence of cracks and particle porosity play key roles in increasing the penetration depth, and as these characteristics are more pronounced in HPGR-crushed particles, the conversion front clearly penetrates more deeply in these. As can be seen from the trend line equations, the correlation between the penetration depth $\left(P_{d}\right)$ and particle radius $(R)$ can be represented as a power law as per Eq. (1):

$$
p_{d}=\mathrm{aR}^{\mathrm{b}} \quad \text { Eq. }(1)
$$

With $b=0.7-0.8$ more or less the same for the products of the two different comminution devices, the only difference is the value of a, which is 1.4 for the particles crushed using HPGR and 0.8 for particles crushed using cone crusher. The exponential relationship could be related to the mechanics of the crack propagation, with the exponent $b$ as a material related parameter (since it is more or less the same for both sets) and the parameter a related to the comminution mode.

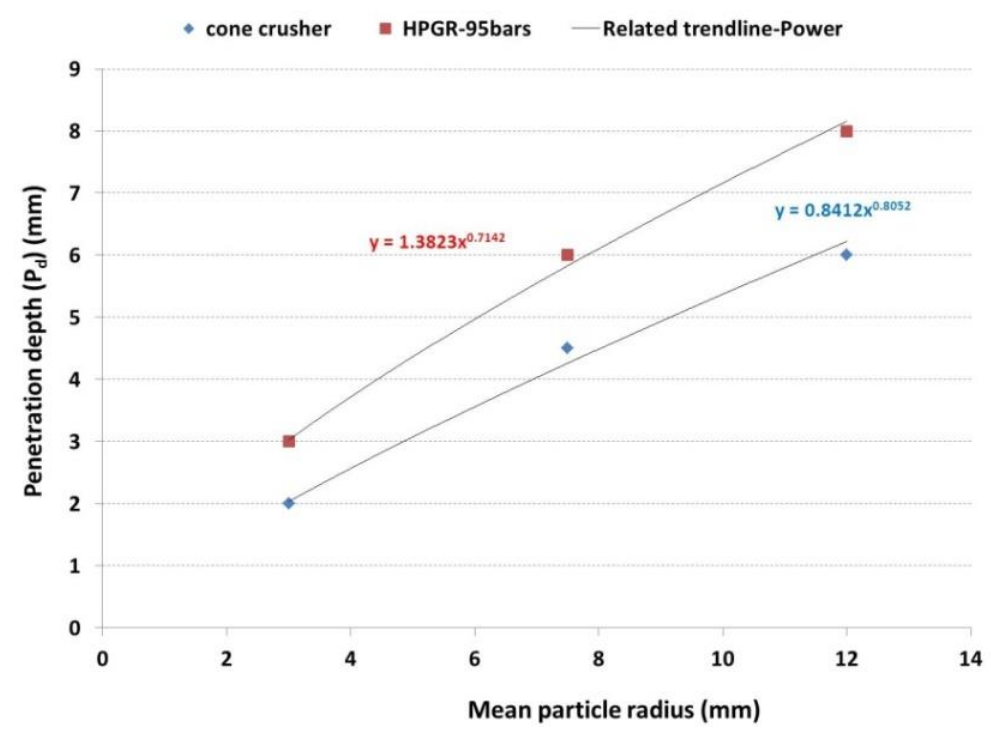

Figure 6: The penetration depth $\left(\mathrm{Pd}_{\mathrm{d}}\right)$ vs mean particle radius in the HPGR-95 bars and cone crusher product. 


\section{Fitting data with models}

The following analysis refers to the $\mathrm{Zn}$ extraction data generated in as set of bioleaching experiments in circulating flow fixed-bed reactors extending over 11-months as described by Ghorbani et al. (2012a). The analysis focuses only on the active bioleaching phase and does not consider the first 50 days of treatment ( 30 days of acid leaching and 20 days for adaptation of micro-organisms after inoculation).

\subsection{Shrinking Core Model}

Figure 7 shows the application of the diffusion controlled shrinking core model $\left(1-2 / 3 X-(1-X)^{2 / 3} v s . t\right)$ and surface reaction controlled shrinking sphere model $\left(1-(1-X)^{1 / 3}\right.$ vs. $\left.t\right)$ to the leaching of zinc in the leach liquors from the different reactors. The results clearly show that leaching from large particles contrary to the common assumption - does not follow either of the two models.

From the profiles in Figure 7 one can discern three different stages during the leaching process. The first stage is leaching of grains at the surface of the ore particles that extends over the first 50 days, followed by a second stage, which is leaching of the grains located in the subsurface within the thickness of the penetration depth. The curves in Figure 7 indicate a clear inflection point, after which the slope changes. This point lies between days 90 and 120 for the HPGR-crushed particles (with larger particles tending to show the inflection later) and between days 120 and 150 for the cone-crushed material. It is postulated that this point relates to the depletion of mineral grains directly accessible from the surface of particles (via cracks); thereafter reagent has to penetrate deeper through micro-pores.

Thus in the cracked rim, there is a rapid penetration of leaching reagent through the crack network, but slow leaching from zones between cracks. This is illustrated in Figure 8. This mechanism would also explain why the HPGR profiles in Figure 5 are quite 'flat', showing rapid penetration to a certain depth, but only slow and quite homogeneous leaching thereafter. Cone-crushed particles, which have few cracks (as indicated in Table 4) would not show this behaviour.

This second stage is a combination of diffusion and reaction, which proceeds up to a certain penetration depth. After depletion of mineral grains that can be accessed by this second stage process, a much slower mechanism governs leaching from the inner core. This is shown schematically in Figure $8 \mathrm{~b}$. It should be noted that there is not enough data for this third phase leaching as the experiments were not continued for long enough. It is thus not possible to determine what mechanism this follows, but the trends in Figures 1 and 2 indicate that the last stage of 
leaching can be approximated as linear. Given the time-scale of a typical heap leaching process, a linear model to describe this slow residual leaching is acceptable.
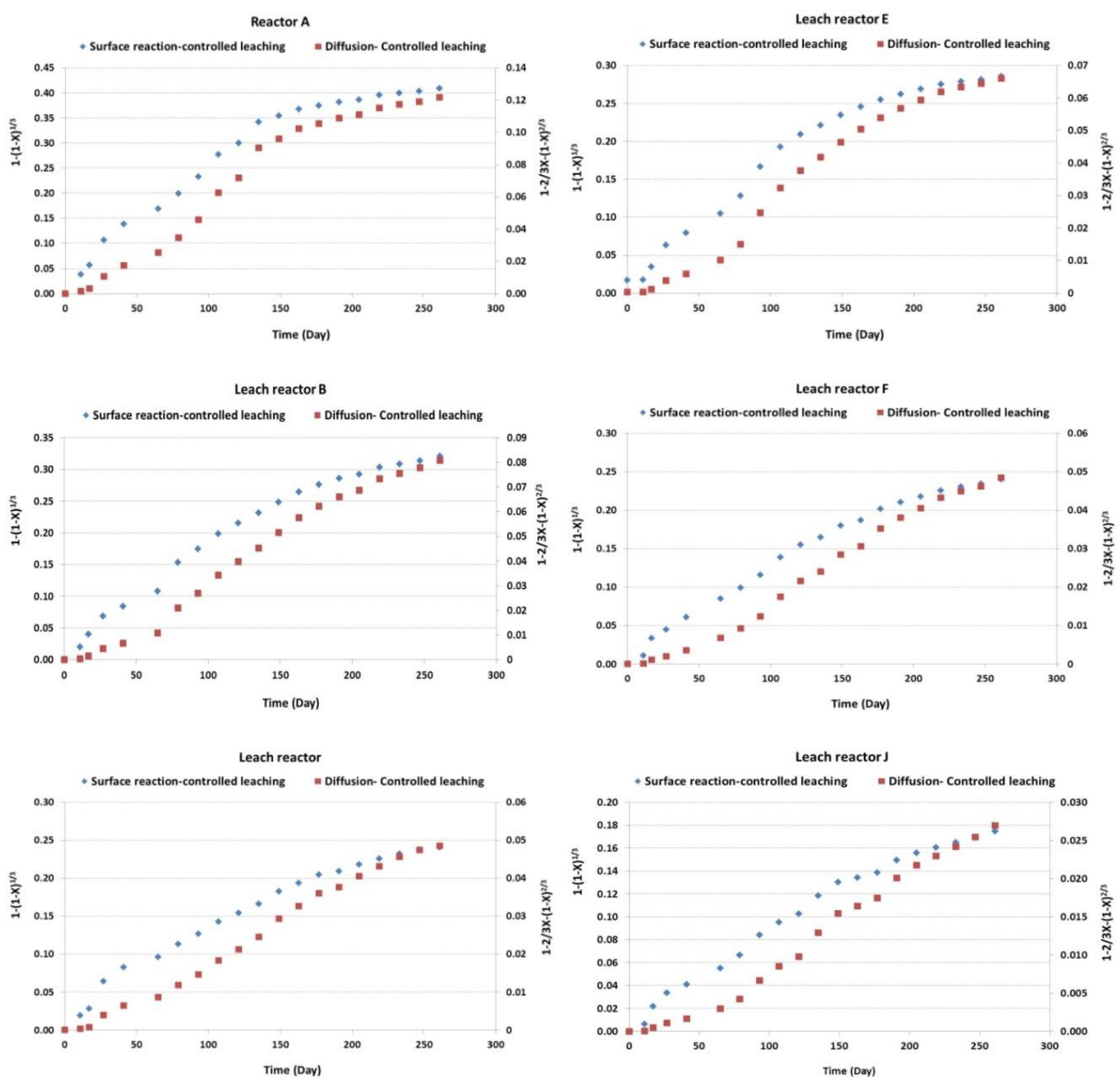

Figure 7: Application of the shrinking core model and shrinking sphere model to the leaching of zinc in the leach liquors from the reactors A (HPGR-95bars-Small size fraction), E (cone crusher-Small size fraction), B (HPGR-95bars-Medium size fraction), F (cone crusher-Medium size fraction), C (HPGR-95bars-large size fraction) and $J$ (cone crusher-large size fraction). 

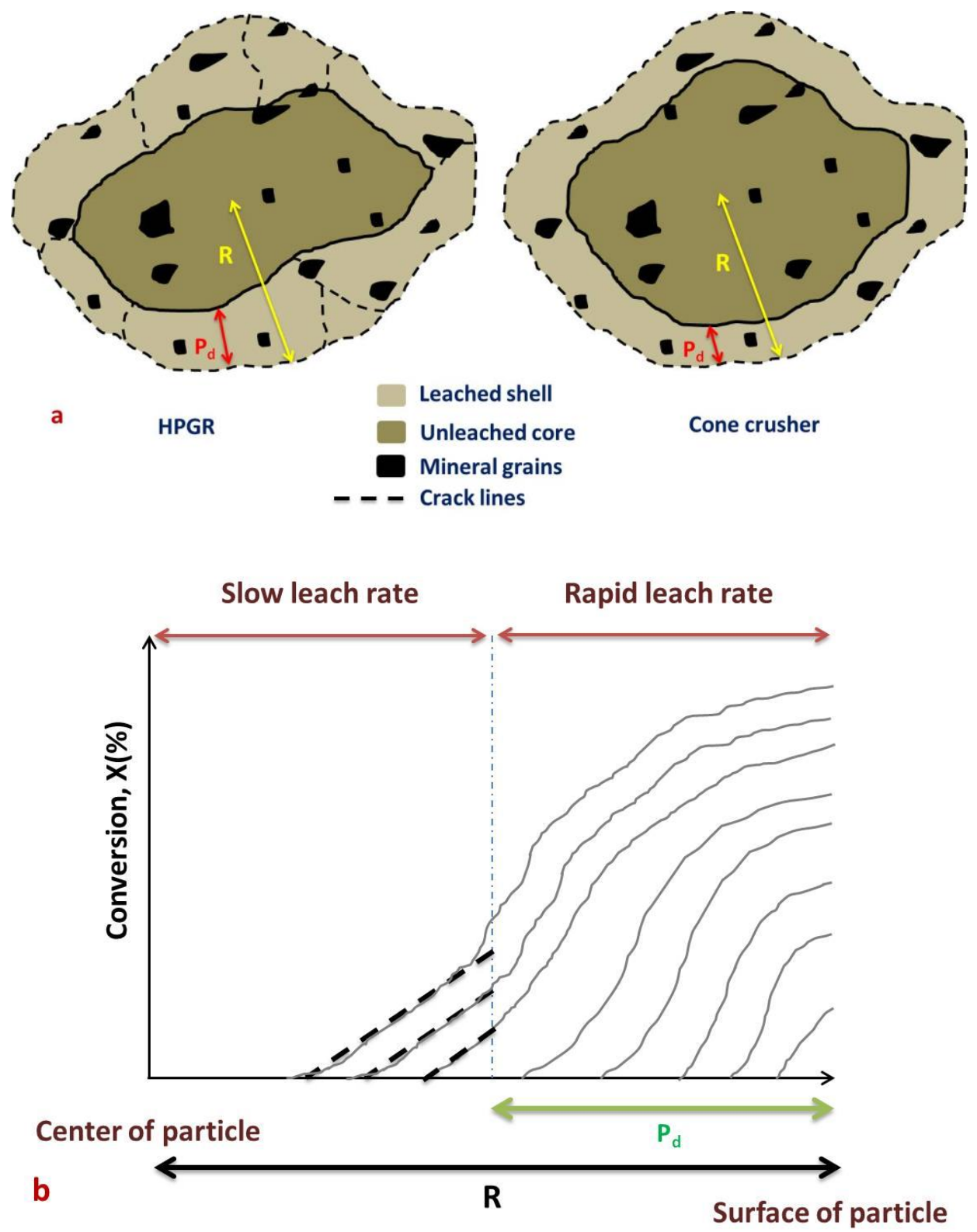

Figure 8: Schematic cross section of a large particle, a. partially leached particle with the effective penetration depth $\left(P_{d}\right)$, b. two different leach regime.

\subsection{Reaction-diffusion Model}

As discussed, the second stage of the leaching process is a combination of diffusion and reaction. Modelling a simplified reaction-diffusion system in spherical coordinates and how it fits with experiments data will be discussed here. For this, consider a porous, structurally uniform, spherical ore particle of radius $\mathrm{R}$ which is submerged in a lixiviant solution, and which contains small amounts of solid reactant evenly distributed along the pore walls. The solid reactant $\left(\mathrm{B}_{(\mathrm{s})}\right)$, is dissolved by a single reagent $\left(A_{(a q)}\right)$ according to the formula (2): 


$$
A_{(a q)}+B_{(s)} \rightarrow \text { dissolved products }
$$

Since every reaction within the particle involves the consumption of reagent $A$, under these conditions, the transient simultaneous diffusion-reaction of species A through a spherical domain is described by Eq. (3) (Dixon, 1996):

$$
D_{A e}\left[\frac{\partial^{2} C_{A}}{\partial r^{2}}+\frac{2}{r} \frac{\partial C_{B}}{\partial r}\right]+S_{A}=\frac{\partial C_{A}}{\partial t}
$$

where $D_{A e}$ is the effective diffusivity of reagent $A$ within the particle pores. It should be noted that $C_{A}$ is the volumetric concentration of reagent $A$ in solution and $C_{B}$ is the volumetric concentration of the solid $B$ in the particle. To convert to a mass concentration, the mean particle density needs to be introduced, but this is ignored here for simplicity of the discussion. For the source term $\mathrm{S}_{\mathrm{A}}$, the reaction of solute $A$ with solid $B$ is assumed to follow simple first order kinetics in terms of both $A$ and B, i.e.

$$
S_{A}=\left(\frac{1-\varepsilon}{\varepsilon}\right) \frac{d C_{B}}{d t}=-k C_{A} C_{B}
$$

Where $\mathrm{k}$ is the corresponding rate constant and $\varepsilon$ represents the porosity of the solid particle. The following boundary conditions apply:

$$
\begin{aligned}
& r=0: N_{A}=-D_{A e} \frac{\partial C_{A}}{\partial r}:_{r=0}=0 \\
& r=R: N_{A}=-D_{A e} \frac{\partial C_{A}}{\partial r}:_{r=R}=k_{C}\left(C_{A R}-C_{A b}\right)
\end{aligned}
$$

where $k_{C}$ is the corresponding rate constant, $C_{A R}$ is the concentration of the reagent $A$ at the particle radius $R$ and $C_{A b}$ is the concentration of the reagent $A$ in the bulk solution external to the particles . This system can be solved with a simple central difference numerical scheme.

Figure 9 shows comparison of the measured conversion $X(Z n)$ and simulated conversion $X(Z n)$ over time from leach reactor A (HPGR-95bars-small size fraction) and leach reactor J (cone crusher-large size fraction), which represent the two most extreme cases in the present study. As it can be seen, there is good agreement between the trends predicted by the first order particle diffusion-reaction model and the average conversion $X(Z n)$ over time from different positions within the ore particle evaluated with the X-ray CT. The key difference in the model parameters chosen for the two scenarios relate to porosity and the value of the Damkohler number, which represents the relative effects of reaction and diffusion phenomena. The Damkohler number for the cone-crushed particles is significantly lower, indicating diffusion effects are more dominant, but in either case both effects have an influence on the dissolution mechanism. 
The rigorous reaction-diffusion model was not pursued further due to its computational complexity and the fact that it is based on certain over-simplified assumptions, such as homogeneous porosity, structurally uniform, spherical ore particle, and it ignores the inner core into which cracks do not penetrate.
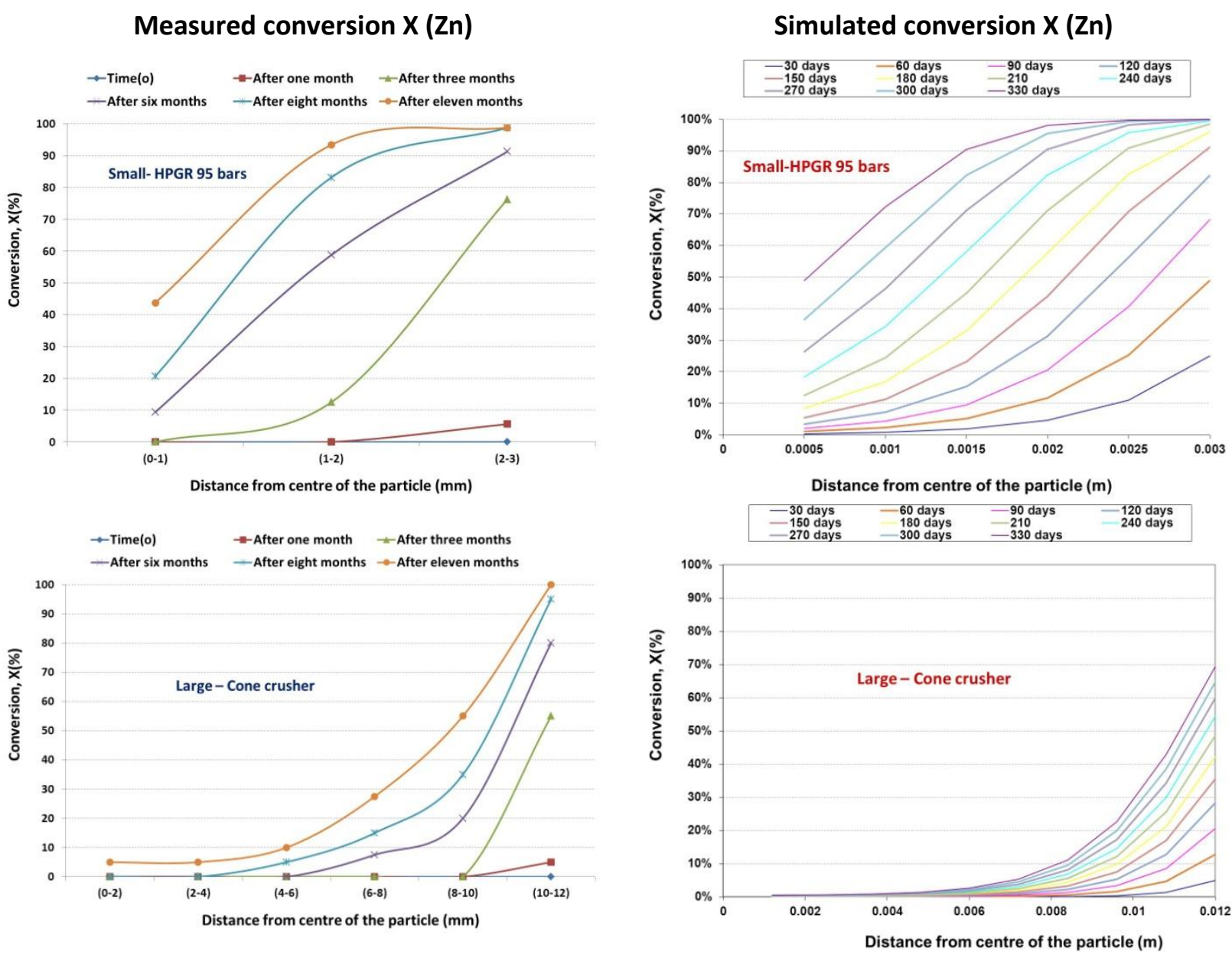

Figure 9: Comparison of the measured conversion $X(Z n)$ and simulated conversion $X(Z n)$ over time from leach reactor A (HPGR-95bars-small size fraction) and leach reactor J (cone crusher-large size fraction).

\section{$3.3 \quad$ K- $\varphi$ Model}

Although the explicit diffusion-reaction model can capture the key reaction trends reasonably well, it is for modelling purposes somewhat cumbersome, as it would require solution of the set of equations (7) to (10) for all particle size classes at each time step. As a simplification, the intrinsic 
kinetics of leaching can generally be described by a rate expression of the following form (Bouffard, 2003; Bouffard and Dixon, 2001):

$\frac{d X}{d t}=k(T) \cdot f\left(c_{i}\right) \cdot w(1-X) \quad$ Eq. (7)

where $k\left(T, d_{0}\right)$ is a rate constant, which is a function of temperature (i.e., Arrhenius's law) and initial mineral grain size (or surface area), $f(C)$ is a function of solution composition, accounting for the effect of concentration of one or several reagents, for example first or second order relationships. $\mathrm{w}(1-\mathrm{X})$ is a function of the fraction of unreacted mineral, which represents the changing topology of the mineral surface over the course of leaching. Often, for finely ground particles, $w(1-X)$ conforms to some well-known form, such as the shrinking sphere or shrinking core model. A simple generalised form for the topological rate term can be given as follows (Bouffard, 2003):

$w(1-X)=k(1-X)^{\varphi} \quad$ Eq. (8)

where $\varphi$ is equal to $2 / 3$ in the case of the shrinking sphere model but may be as high as 3 when the distribution of effective grain size is particularly wide. For each particle size fraction, Eq. (7) can be written as the following equation, assuming temperature, particle size and chemical condition are all fixed, as they would be in the present reactor experiments:

$\frac{d X}{d t}=\mathrm{K} \cdot(1-X)^{\varphi} \quad$ Eq. (9)

where $\mathrm{K}$ is the corresponding rate constant. Eq. (9) can be integrated to yield:

$\mathrm{X}(\mathrm{t})=1-(1+\mathrm{K}(\varphi-1) \mathrm{t})^{\frac{1}{1-\varphi}} \quad \varphi \neq 1 ; \quad \mathrm{t}_{\text {lim }}=\frac{1}{\mathrm{~K}(1-\varphi)} \quad$ if $\varphi<1 \quad$ Eq. (10)

If $\varphi<1$, the function $X(t)$ will reach the value 1 after a finite time, $t_{\text {lim }}$, as indicated. For any values of $t$ larger than this limiting time, $X(t)=1$, and the function defined above is no longer valid. For $\varphi>1$, $X(t)=1$ for $t \rightarrow \infty$.

Table 4 shows $K$ and $\phi$ values obtained by minimisation of mean squared error (MSE) using Eq. (10), for the selected particles from different size fractions crushed using HPGR-95 bars and cone crusher. 
Table 4: Obtained $K$ and $\varphi$ values for leach reactors from different size fractions crushed using HPGR-95 bars and cone crusher.

\begin{tabular}{l|ccc|c|c}
\hline \multirow{2}{*}{ Size fraction (mm) } & \multicolumn{2}{|c|}{ K $\left(\right.$ day $\left.^{-1}\right)$} & \multicolumn{2}{c}{$\boldsymbol{\varphi}$} \\
\cline { 2 - 5 } & HPGR & cone crusher & HPGR & cone crusher \\
\hline Small (-6.75+5.25) & 0.012 & 0.007 & 1.59 & 1.88 \\
Medium (-16+14) & 0.007 & 0.005 & 1.65 & 2.00 \\
Large (-25+23) & 0.006 & 0.003 & 2.45 & 2.15 \\
\hline
\end{tabular}

As given in Table 4, in both comminution devices the value for $\mathrm{K}$ decreases with increasing particle size. This was expected; since K represents the initial (surface) rate and with increasing particle sizes, the specific surface area declines. In all three sizes classes the value of $\mathrm{K}$ is higher for those particles that were crushed using HPGR, than for those crushed using a cone crusher, which indicates the larger effective initial surface in the particles crushed using HPGR. Figure 10 shows the relationship between $\mathrm{K}$ and mean particle radius $(\mathrm{R})$. According to the trend lines, the correlation between $\mathrm{K}$ and particle radius ( $R$ ) can be represented by a power law as per Eq. (11):

$$
K=\mathrm{cR}^{\mathrm{d}} \quad \text { Eq. (11) }
$$

With the parameter $d \approx-0.5$ more or less the same for the products of the two different comminution devices, whereas the only difference is the value of $c$, which is 0.021 for the particles crushed using HPGR and 0.014 for particles crushed using a cone crusher. The difference in the K values is due to the specific surface area in the different size fractions and created through the different comminution devices. In this context, it is interesting to note that the ratios of values of a in Eq. (1) ( $a_{H P G R} / a_{c o n e}$ cerusher $\left.=1.6\right)$ relating to penetration depth, and those for the values $c$ in Eq. (11) $\left(\mathrm{C}_{\mathrm{HPGR}} / \mathrm{c}_{\mathrm{cone}}\right.$ cerusher $\left.=1.5\right)$ relating to the kinetic constant compare closely, suggesting that the rate parameter $\mathrm{K}$ is indeed closely linked to the crack network created by the different comminution devices. 


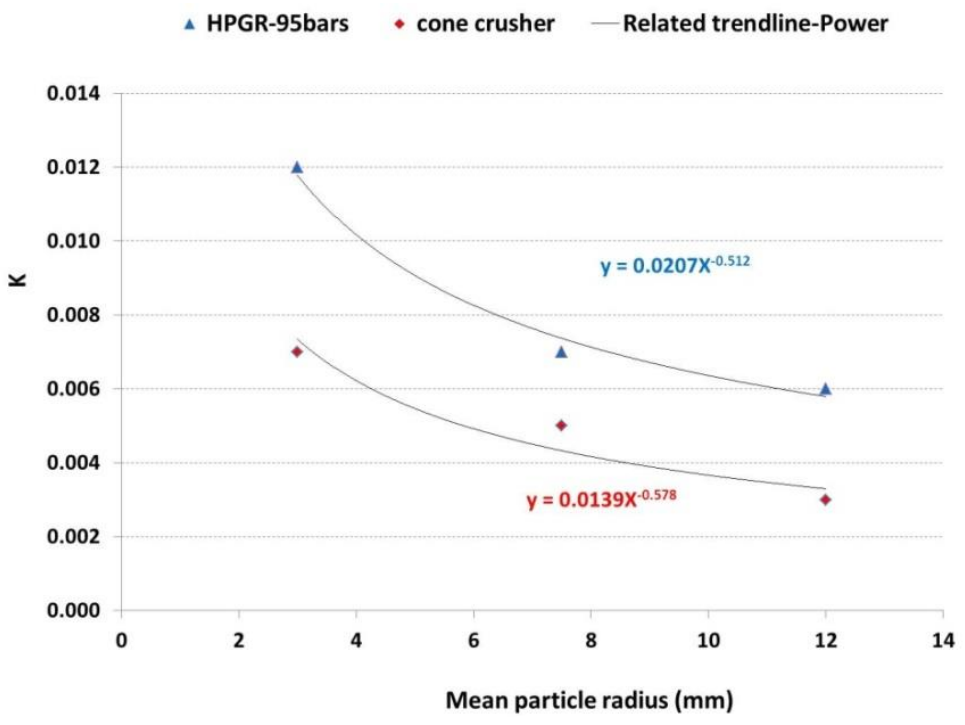

Figure 10: Relationship between $\mathrm{K}$ and mean particle radius.

Figure 11 shows a comparison of the conversion $X(Z n)$ for the leach reactors vs. time for experimental data and the fitted curve obtained for $\mathrm{K}$ and $\varphi$ values from different size fractions. 
HPGR
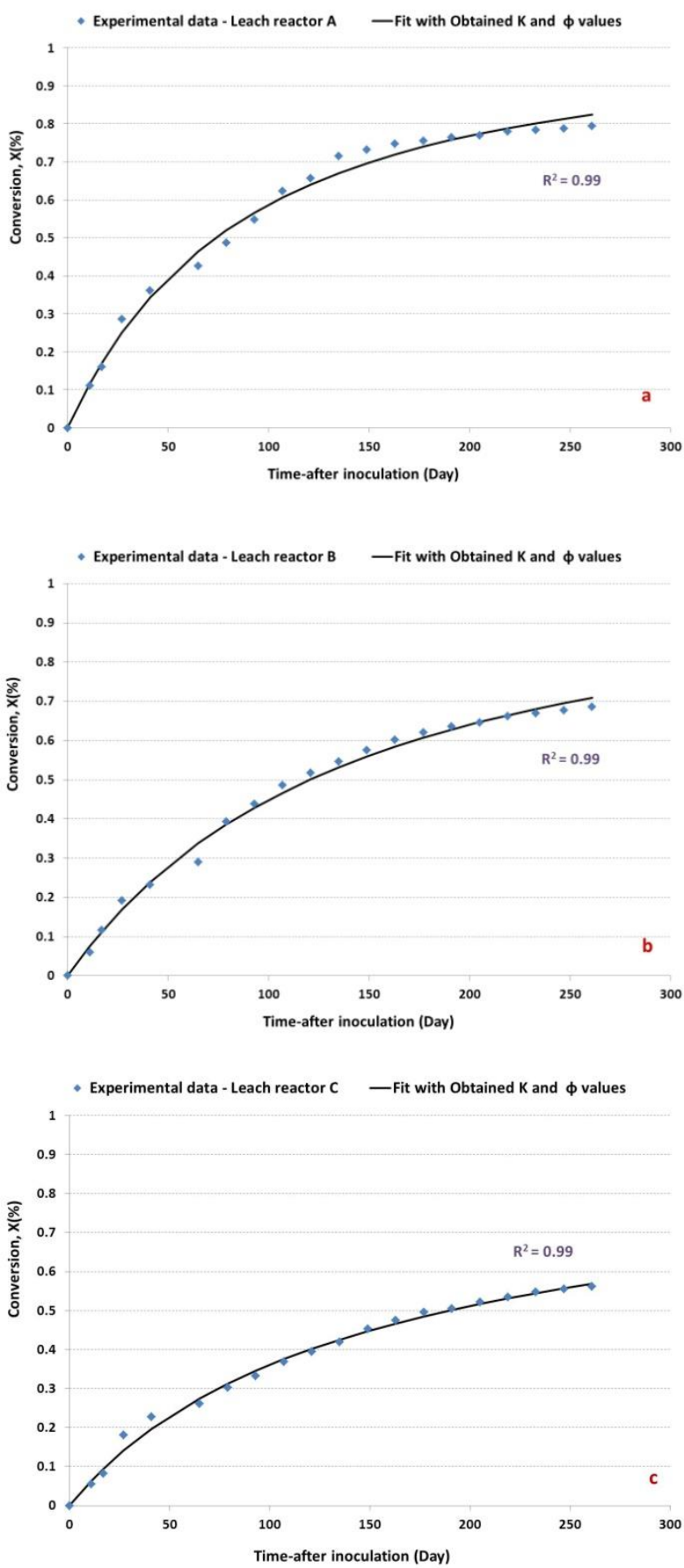

cone crusher
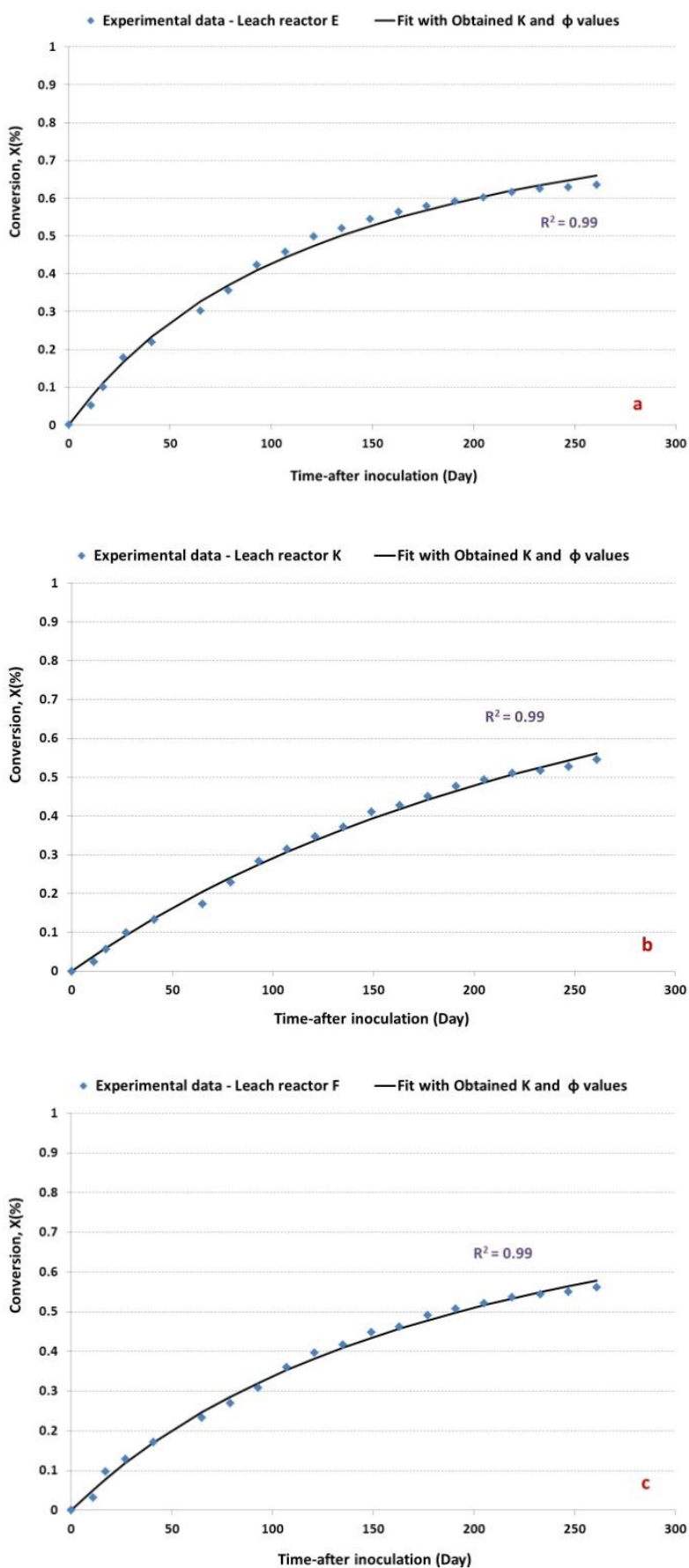

Figure 11: Comparison of the conversion $X(Z n)$ vs. time for experimental data and fit curve for obtained $\mathrm{K}$ and $\boldsymbol{\varphi}$ values for leach reactors from different size fractions crushed using HPGR-95bars and cone crusher (a. Small, b Medium and c. Large). 


\subsection{Extended K- $\varphi$ Model}

As discussed, in fitting data with the shrinking core and shrinking sphere models there are clearly three different stages during the leaching process - leaching of grains at the surface, leaching up to penetration depth and very slow leaching phase from the core. While the fits shown in Figure 11 are generally good, there is clearly an indication that modelled curve and experimental data begin to diverge from each other towards the end of the leach, where the proposed third phase - deep particle leaching - becomes dominant. The model over-predicts the rate of leaching, which, although marginal here, could lead to significant mis-predictions of the rate of leaching from large particles in the long run.

Therefore, an alternative analysis was considered in extension of the model presented in Eq. (10). Here it is assumed that there is a readily leachable fraction $(\alpha)$ of $\mathrm{Zn}$ in the ore, which is accessible through the pore network, and a poorly leachable fraction $(1-\alpha)$, which is locked within zones that are not cracked. The readily leachable fraction $(\alpha)$ varies with particle size and comminution method and is likely to be related to the penetration depth $\left(P_{d}\right)$ discussed earlier. In this case, the general topology model needs to be adjusted such that it applies only to the leachable fraction $(\alpha)$ as follows:

$$
\frac{d X}{d t}=\mathrm{K} \cdot\left(1-\frac{X}{\alpha}\right)^{\varphi}
$$

Where $\mathrm{X}$ remains the overall fractional $\mathrm{Zn}$ conversion (i.e. relative to total $\mathrm{Zn}$ content). This equation can be integrated to yield:

$X(t)=\alpha\left[1-\left(1+(\varphi-1) \frac{K t}{\alpha}\right)^{\frac{1}{1-\varphi}}\right] \quad \varphi \neq 1 ; \quad t_{l i m}=\frac{\alpha}{K(1-\varphi)} \quad$ if $\quad \varphi<1$

where the limiting time $t_{\lim }$ applies for $\varphi<1$, as discussed for Eq. (10).

Finally, after depletion of the leachable fraction $(\alpha)$, the reaction proceeds further at a very slow rate, probably related to the solid-state diffusion of reacting species, which occurs at diffusivities orders of magnitude smaller than that assumed for a porous matrix. From the data in Figures 1 and 2 , it appears that leaching towards the end proceeds in a more or less linear fashion, and to account for this in the model, a simple linear term is added to above equation:

$$
X(t)= \begin{cases}\alpha\left[1-\left(1+(\varphi-1) \frac{K t}{\alpha}\right)^{\frac{1}{1-\varphi}}\right]+\beta t & t \leq \frac{\alpha}{K(1-\varphi)} \text { if } \varphi<1 ; \quad \text { all } t \text { if } \varphi>1 \\ \alpha+\beta t & t>\frac{\alpha}{K(1-\varphi)} \quad \text { if } \varphi<1\end{cases}
$$


Table 5 shows $K, \varphi, \alpha$ and $\beta$ values obtained by minimisation of mean squared error (MSE) using Eq. (14), for the leach reactors data from different size fractions crushed using HPGR-95 bars and cone crusher.

Table 5: Obtained K, $\boldsymbol{\varphi}, \alpha$ and $\beta$ values for leach reactors data from different size fractions crushed using HPGR95 bars and cone crusher.

\begin{tabular}{c|c|c|c|c|c|c|c|c|}
\hline \multirow{2}{*}{ Size fraction } & \multicolumn{2}{|c|}{$K$ (day $\left.^{-1}\right)$} & \multicolumn{2}{c|}{$\varphi$} & \multicolumn{2}{c|}{$\alpha$} & \multicolumn{2}{c}{$\beta$ (day $\left.^{-1}\right)$} \\
\cline { 2 - 10 } & HPGR & $\begin{array}{c}\text { cone } \\
\text { crusher }\end{array}$ & HPGR & $\begin{array}{c}\text { cone } \\
\text { crusher }\end{array}$ & HPGR & $\begin{array}{c}\text { cone } \\
\text { crusher }\end{array}$ & HPGR & $\begin{array}{c}\text { cone } \\
\text { crusher }\end{array}$ \\
\hline Small & 0.011 & 0.005 & 0.793 & 0.217 & 0.789 & 0.418 & $0.00 \mathrm{E}+00$ & $8.64 \mathrm{E}-04$ \\
Medium & 0.007 & 0.003 & 0.793 & 0.284 & 0.643 & 0.322 & $1.79 \mathrm{E}-04$ & $9.30 \mathrm{E}-04$ \\
Large & 0.005 & 0.002 & 0.710 & 0.252 & 0.565 & 0.225 & $1.15 \mathrm{E}-06$ & $8.10 \mathrm{E}-04$ \\
\hline
\end{tabular}

Comparing Table 5 to the Table 4 shows that the values for $\mathrm{K}$ remain roughly the same and follow the same trends as shown in Figure 12. This is expected, as K describes the initial slope of the leach curves, which remain the same regardless of the model used. Thus $\mathrm{K}$ can still be modelled with Eq. (11), with the model parameters of $d \approx-0.5$ almost the same for the products of the two different comminution devices, and c, which is 0.019 for the particles crushed using HPGR and 0.009 for particles crushed using a cone crusher.

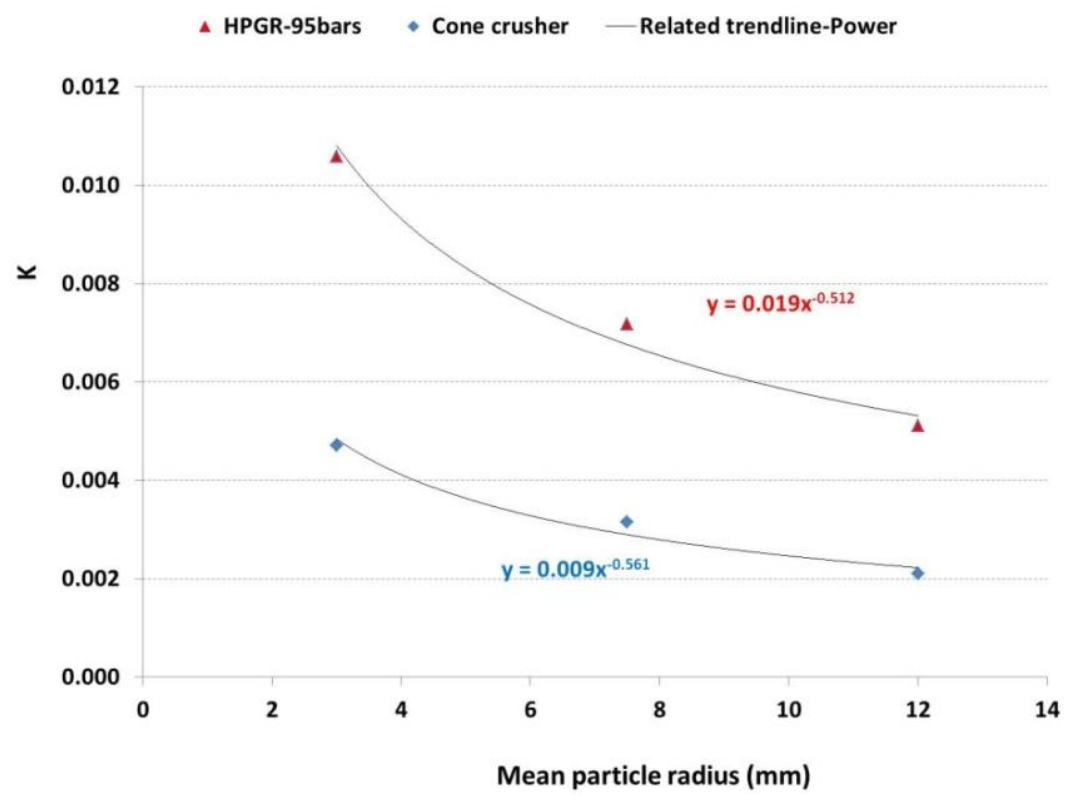

Figure 12: Relationship between $\mathrm{K}$ and mean particle radius. 
The values for $\varphi$ completely change with the introduction of the readily leachable fraction $(\alpha)$ and always are below 1 . As given in Table 5, $\varphi$ does not significantly change with particle size and can directly be related to crushing mode. The small value of $\varphi$ for cone crusher indicates that the leaching is nearly linear, i.e. that exposed mineral is mostly near the surface, which relates to the much lower $\alpha$ values found for this comminution mode.

Figure 13 shows the relationship between the readily leachable fraction $(\alpha)$ and fractional penetration volume $\left(P_{d} / R\right)^{3}$, which could be perceived as the volumetric portion of the particle that is accessible to reagents. As the trend line shows, the correlation follows almost perfect linear fits, further confirming the link between the penetration depth and leach behaviour. Leachable fraction ( $\alpha$ ) can thus be described as per Eq. (15):

$$
\alpha=\alpha_{0}+\alpha_{1}\left(\frac{p_{d}}{R}\right)^{3}
$$

and the connection between $P_{d}$ and $R$ is described in Eq. 1 .

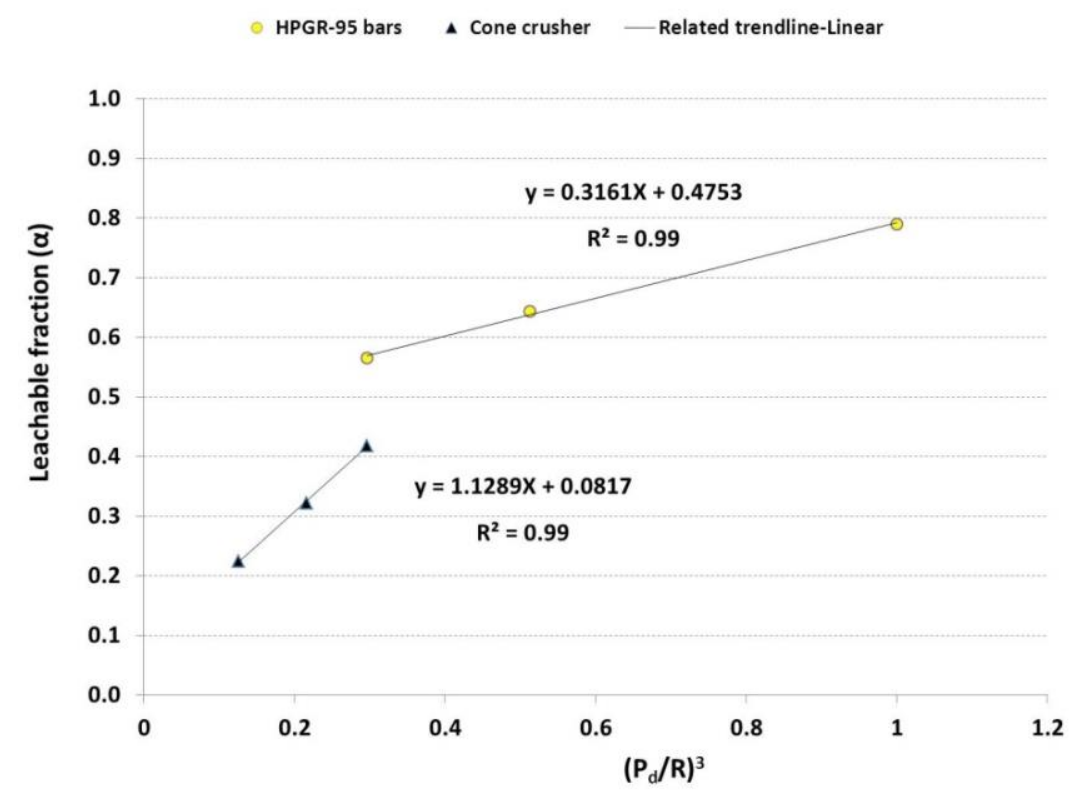

Figure 13: The relationship between the readily leachable fraction $(\alpha)$ and fractional penetration volume $\left(\mathrm{Pd}_{\mathrm{d}} / \mathrm{R}\right)^{3}$.

The values close to zero for $\beta$ in Table 5 indicate that after depletion of the leachable fraction $(\alpha)$, the reaction proceeds further at a very slow rate, which is almost negligible. This indicates that metal 
extraction from the uncracked zones within the particles takes a very long time and probably follows a solid-state diffusion mechanism. In terms of extraction in a heap leaching context this portion of leaching from large particles is essentially beyond economic recovery and exact description would not be critical

Figure 14 shows a comparison of the conversion X (Zn) for leach reactors vs. time for experimental data and the fitted curve obtained using the extended $\mathrm{K}-\varphi$ model. As can be seen, the fit is now near perfect compared to Figure 10. However, more significant is the fact that in the extended model all model parameters can be correlated to the two fundamental variables studied - particle size and crushing mode. 
HPGR
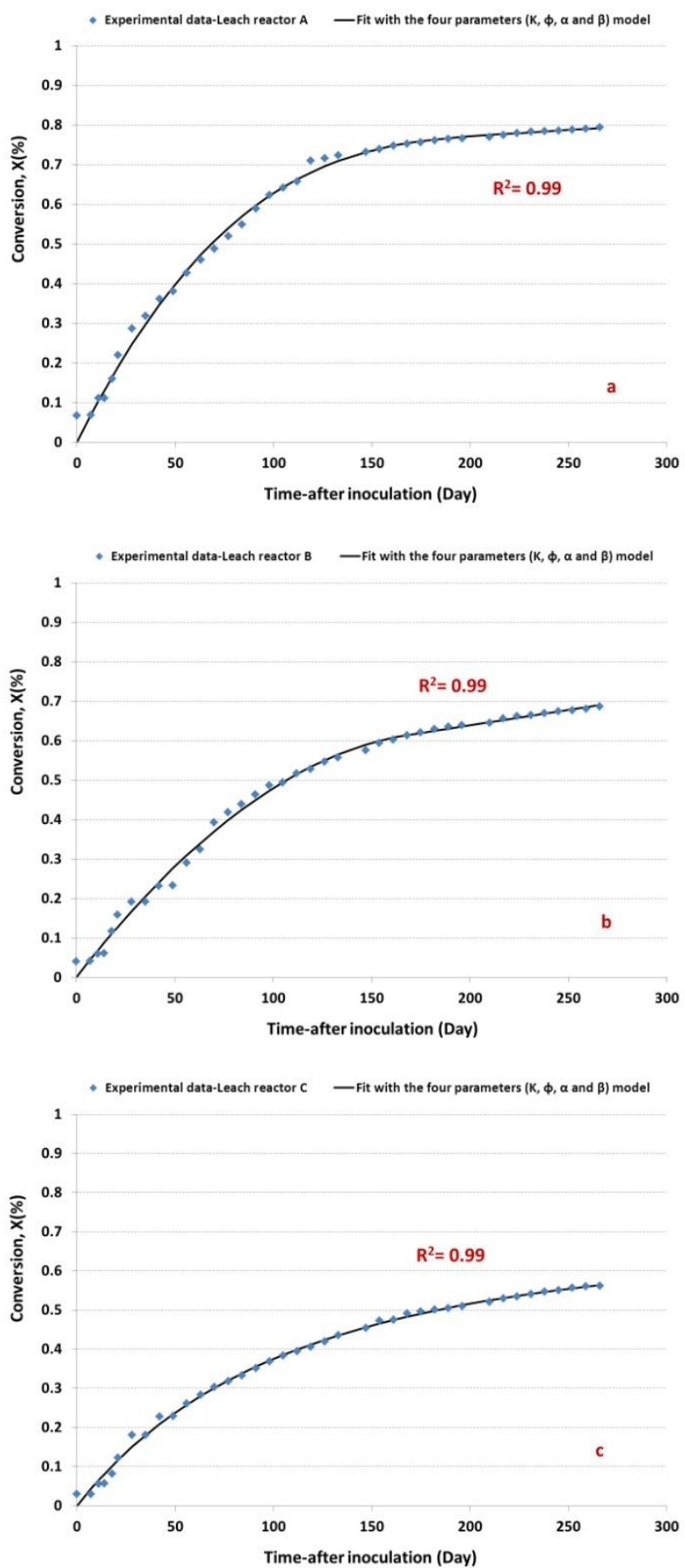

Cone crusher
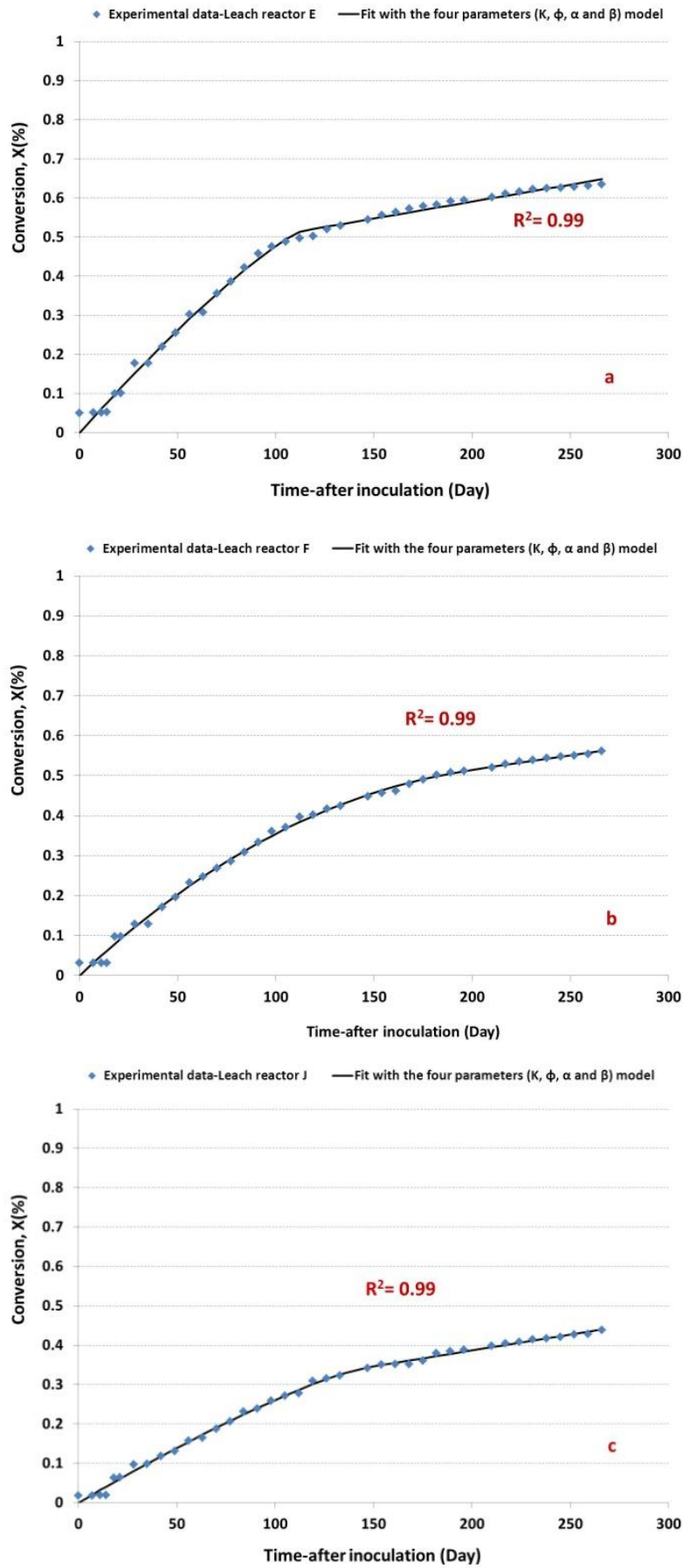

Figure 14: Comparison of the conversion X (Zn) vs. time for experimental data and fit curve of 4-parameter model with obtained $K$ and $\boldsymbol{\varphi}$ values for leach reactors from different size fractions crushed using HPGR-95bars and cone crusher (a. Small, b Medium and c. Large). 


\subsection{Model Summary and Discussion}

Following the leaching process within the selected particles from different size fractions with X-ray $\mathrm{CT}$ and other techniques provided a clear image of the effective parameters in leaching from large particle in course of heap leaching. Table 6 summarizes the core model parameters from Eq. (14) and how they relate to the other variables, namely the comminution mode, the penetration depth $\left(P_{d}\right)$ and mean particle radius $(R)$. In this analysis only comminution mode and particle radius remain as true variables in a heap leach system, which makes modelling of the reaction process straightforward.

Table 6: Final rate model (Eq. (13)) and summary of the core model parameters. Note mean particle radius (R) is in $[\mathrm{mm}]$.

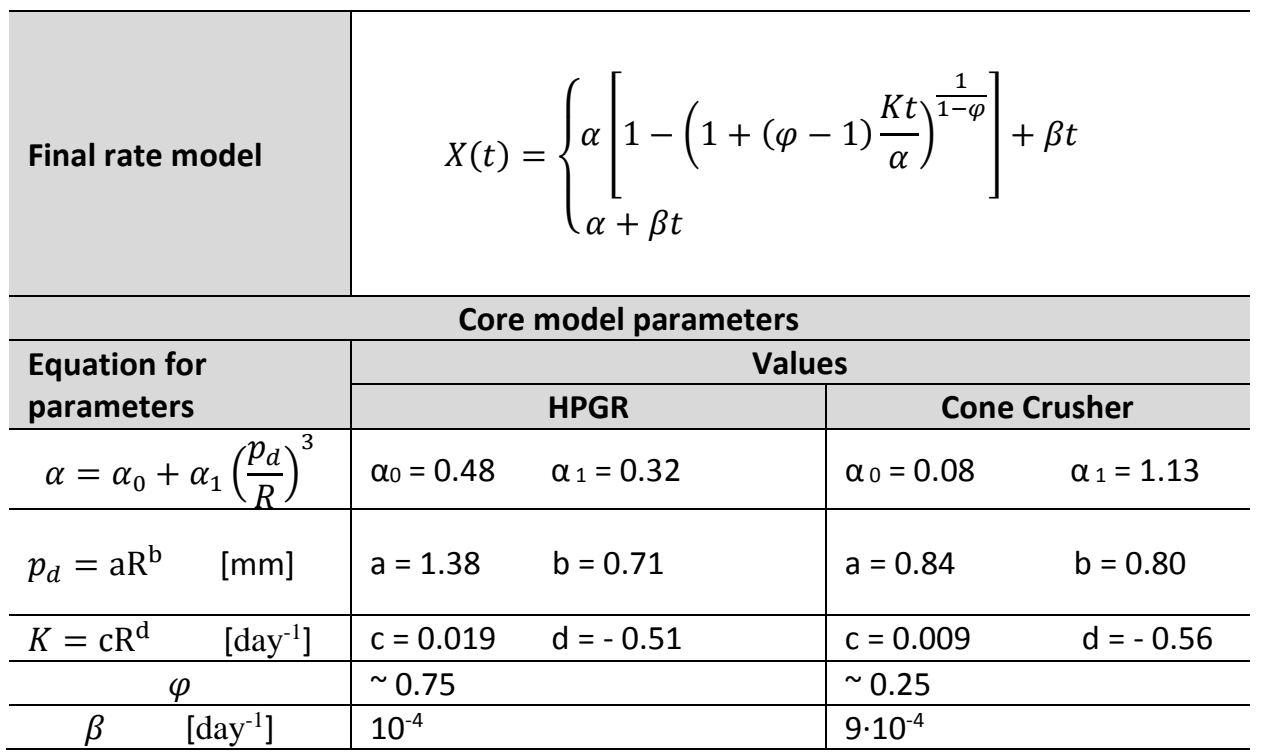

The derivation of the various model parameters listed in Table 6 follows from a systematic analysis of the progression of leaching overall and within individual particles. It has clearly shown that leaching rate and extent are linked to the mode of comminution via the formation of a network of cracks and micro-pores in the outer rim of the particle. The penetration depth $\left(P_{d}\right)$ of this network can be related to particle size via a power relationship with the exponent very similar for the two methods tested here, which indicates a connection to the mechanics of crack formation. Total pore space density within the cracked rim could not be determined, but appears to relate closely to the model parameter $\varphi$, which represents the 'remoteness' of mineral grains from the nearest crack or pore. Again it is interesting to note that the value of $\varphi$ is more or less constant for the two comminution methods, independent of particle size, but substantially different between the two 
methods. This points to a very different nature of the cracks formed by the two methods: Relatively deep cracks with few micro-fissures created by HPGR; and a denser network of micro-fissures with few cracks near the surface created by the cone crusher. This is also borne out by both physical observation and pore size analysis presented in Ghorbani et al., 2012b.

This interpretation is further supported by the leachable fraction parameter $(\alpha)$, which shows a direct relationship with the relative particle volume of the cracked $\operatorname{rim},\left(p_{d} / R\right)^{3}$. This correlation is much stronger for the cone crusher than the HPGR (the value of $\alpha_{1}$ is 3.5 times larger), which would make sense if the former mode resulted in a dense network of micro-pores as opposed to a less dense network of larger cracks for the latter. However, the tools available to this study did not allow a closer characterisation of the nature and extent of micro-pores created by the two different comminution methods.

Finally, the nature of 'residual' leaching from the un-cracked portion of particles merits further exploration. While the leach data generated in the present study was insufficient to characterise this more closely, the extremely slow rates estimated $\left(\beta<10^{-3}\right.$ day $^{-1}$ for the cone crusher and $<10^{-4}$ day $^{-1}$ for the HPGR material) point to a process with a rate constant several orders of magnitude slower than the principal reaction-diffusion processes studied here. It is well possible that this could relate to solid-state diffusion phenomena through zones, which have remained unaffected by any cracks or micro-fissures. Careful long-term studies could reveal more about this process, but from a practical heap leaching point of view, this mode of leaching refers to the portion of mineral that is unlikely to be recovered economically. The principal focus must therefore remain on preparing an ore charge, which minimises the occurrence of this slow-leaching phase.

\section{CONCLUSION}

Investigation of mineral conversion within single ore particles through $\mathrm{X}$-ray $\mathrm{CT}$ indicated that leaching from large particles leads to near complete conversion near the surface, but only partial conversion in the grains that are closer to the centre of particles. After all minerals near the surface are depleted, the leaching regime changes from the readily accessible grains on the particle surface ore exposed to large cracks to the more inaccessible grains within particles. In the HPGR product, the reaction zone thus covers both the particle surface and a relatively deep subsurface zone, which is connected to the surface through cracks. In the case of the cone crusher product, the outer surface of the particle is the main reaction surface with only limited reaction occurring in the subsurface zones and not nearly as deep as the particles crushed by HPGR. This implies the existence of micro- 
cracks in these particles, which are not detectable by the techniques used in this study. The results indicate that the penetration depth $\left(P_{d}\right)$ is a function of both the comminution mode and the particle size/radius ( $R$ ) and can be described by a similar power-law correlation. It is clear that prevalence of cracks and particle porosity play key roles in increasing the penetration depth, and these characteristics are more pronounced in HPGR-crushed particles.

Application of neither the shrinking core nor the shrinking sphere models is appropriate to describe leaching. Three different stages during the leaching process were identified. The first stage refers to leaching of grains at the surface of the ore particles, followed by a second stage, which is leaching of the grains located in the subsurface within the thickness of the penetration depth. A third stage relates to very slow, linear leaching from uncracked zones within the particle, primarily the inner core.

Application of a simplified reaction-diffusion model in spherical coordinates showed that there is a good agreement between the trends predicted by a first order particle diffusion-reaction model and the average conversion $X(\mathrm{Zn})$ over time from different position within the ore particle evaluated by direct observation using X-ray CT. This analysis also suggests that the progression of leaching is governed by a combination of reaction and diffusion and not solely by one or the other, as implied in the simplified shrinking core/sphere models.

The extended $\mathrm{K}$ - $\varphi$ model that was proposed in Eq. (14) was considered in extension of the intrinsic kinetics equation of the leaching. It is assumed that there is a readily leachable fraction $(\alpha)$ of $\mathrm{Zn}$ in the ore, which is accessible through the pore network and a poorly leachable fraction (1- $\alpha)$, which is locked within zones that are not cracked.

A simple linear term $(\beta t)$ accounts for the very slow rate at which the reaction proceeds after depletion of the leachable fraction $(\alpha)$. The core model parameters can be directly related to just two principal variables, particles size $(R)$ and crushing mode, thus providing a simple, but effective modelling tool which adequately describes leaching from large particles, based on actual, rather than assumed phenomena.

By directly observing the leaching of individual ore particles, and the effects of the crack and pore network on it, this research has provided a sound understanding of the underlying mechanisms of metal extraction. This work has set out a systematic approach to assess a particular ore in this regard and should set the route forward for systematic assessment of an ore towards the design of an efficient heap leach operation to leach it. 


\section{$1 \quad$ ACKNOWLEDGEMENTS}

The authors are grateful to the Centre for Bioprocess Engineering Research (CeBER) (Department of Chemical Engineering, UCT), Paul Keanly (X-Sight X-ray Services), and the Centre for Advanced Scanning Technologies (Department of physic, UCT) for their advice and support. Financial support from the South Africa Research Chair Initiative (SARChI) in Mineral Beneficiation, and a Research Niche Area (RNA) grant from the National Research Foundation (NRF) of South Africa are also acknowledged.

\section{REFERENCES}

Bartlett, R. W., 1992, Simulation of ore heap leaching using deterministic models. Hydrometallurgy, 29, 231-243.

Bouffard, S. C. and Dixon, D. G., 2001, Investigative study into the hydrodynamics of heap leaching processes. Metallurgical and Materials Transactions B., 32, 763-776.

Bouffard, S.C., 2003, Understanding the Heap Biooxidation of Sulfidic Refractory Gold Ores. PhD dissertation University of British Columbia.

Braun, R. L., Lewis, A. E. and Wadsworth, M. E., 1974, In-place leaching of primary sulfide ores: Laboratory leaching data and kinetics model. Metallurgical and Materials Transactions B., 5, 17171726.

D.G. Dixon and J.Petersen: 2003, Comprehensive Modelling Study of Chalcocite Column and Heap Bioleaching; in Copper 2003- Volume VI: Hydrometallurgy of Copper (Book 2), P.A. Riveros, D. Dixon, D. Dreisinger, J. Menacho (eds.), CIM, Montreal, Canada, 493-516.

Davis, G. B. and Ritchie, A. I. M., 1987, A model of oxidation in pyritic mine wastes: part 1 equations and approximate solution. Applied mathematical modelling, 11, 417-422.

Dixon, D. G., 1996, The multiple convolution integral: A new method for modelling multistage continuous leaching reactors, 51, 4759-4767.

Dixon, D. G., 2000, Analysis of heat conservation during copper sulphide heap leaching. Hydrometallurgy, 58, 27-33.

Ghorbani, Y., Becker, M., Mainza, A.N., Franzidis, J-P., Petersen, J., 2011a, Large particle effects in chemical/biochemical heap leach processes - A review, Minerals Engineering, 24, 1172-1184.

Ghorbani, Y., Becker, M., Petersen, J., Mora, S. H., Mainza, A.N, Franzidis, J-P., 2011b, Use of X-ray computed tomography to investigate crack distribution and mineral dissemination in sphalerite ore particles, Minerals Engineering, 24, 1249-1257. 
Ghorbani, Y., Petersen, J., Harrison, S.T.L., Tupikina, O. V., Becker, M., Mainza, A. N., and Franzidis, JP., 2012a, An experimental study of the long-term bioleaching of large sphalerite ore particles in a circulating fluid fixed-bed reactor, Hydrometallurgy, DOI:10.1016/j.hydromet.2012.07.009.

Ghorbani, Y., Mainza, A.N., Petersen, J., Becker, M., Franzidis, J-P., and Kalala, J.T., 2012b, Investigation of particles with high crack density produced by HPGR and its effect on the redistribution of the particle size fraction in heaps, Minerals Engineering, DOI:10.1016/j.mineng.2012.08.010.

Leahy, M.J., Davidson, M.R. and Schwarz, M.P., 2007, A model for heap bioleaching of chalcocite with heat balance: Mesophiles and moderate thermophiles. Hydrometallurgy, 85, 24-39.

Liddell, K. C., 2005, Shrinking core models in hydrometallurgy: What students are not being told about the pseudo-steady approximation, Hydrometallurgy, 79, 62-72.

Miller, G., 2003, Ore geotechnical effects on copper heap leach kinetics. TMS (The Minerals, Metals and Materials Society), 329-342.

Moreno, L., Martinez, J. and Casas, J., 1999, Modelling of bioleaching copper sulphide ores in heaps or dumps. Process Metallurgy, 9, 443-453.

Ogbonna, N., Petersen, J. and Laurie, H., 2006, An agglomerate scale model for the heap bioleaching of chalcocite. The Journal of the South African Institute of Mining and Metallurgy, 106, 433-442.

Pantelis, G., Ritchie, A. I. M. and Stepanyants, Y. A., 2002, A conceptual model for the description of oxidation and transport processes in sulphidic waste rock dumps. Applied Mathematical Modelling, $26,751-769$.

Petersen, J and Dixon, D.G., 2002, Systematic modelling of heap leaching processes for optimisation and design. Proceedings of the EPD Congress and Fundamentals of Advanced Materials for Energy Conversion. TMS, 757-771.

Roman, R.J. and Olsen, C., 1974, Theoretical scale-up of heap leaching. Solution Mining Symposium, AIME, 211-229.

Rossi, G., 1990. Biohydrometallurgy. McGraw-Hill, New York.

Shafer, J.L., White, M.L., and Caenepeel, C.L., 1979, Application of the Shrinking Core Model for Copper Oxide Leaching, Mining Engineering.31, 165-171.

Sidborn, M., Casas, J. Martínez, J. and Moreno, L., 2003, Two-dimensional dynamic model of a copper sulphide ore bed, Hydrometallurgy, 71, 67-81.

Vegliò, F., Trifoni, M., Pagnanelli, F. and Toro, L., 2001, Shrinking core model with variable activation energy: a kinetic model of manganiferous ore leaching with sulphuric acid and lactose. Hydrometallurgy, 60, 167-179.

Velardo, A., Giona, M., Adrover, A., Pagnanelli, F. and Toro, L., 2002, Two-layer shrinking-core model: parameter estimation for the reaction order in leaching processes. Chemical Engineering Journal, 90, 231-240. 